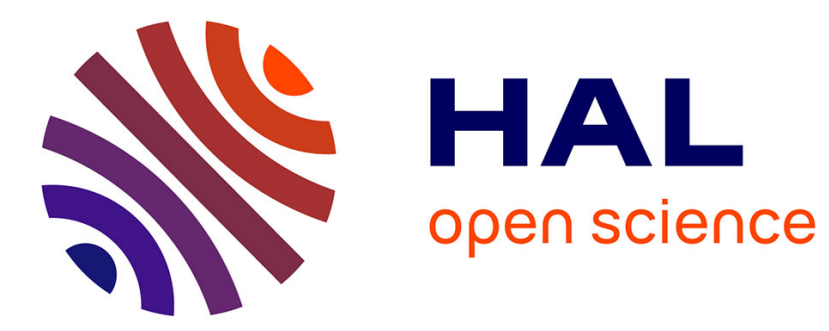

\title{
Numerical analysis of the V-Y shaped advancement flap
}

Djamel Remache, Jérôme Chambert, Julien Pauchot, Emmanuelle Jacquet

\section{To cite this version:}

Djamel Remache, Jérôme Chambert, Julien Pauchot, Emmanuelle Jacquet. Numerical analysis of the V-Y shaped advancement flap. Medical Engineering \& Physics, 2015, 37 (10), pp.987-994. 10.1016/j.medengphy.2015.08.005 . hal-02131277

\section{HAL Id: hal-02131277 \\ https://hal.science/hal-02131277}

Submitted on 16 May 2019

HAL is a multi-disciplinary open access archive for the deposit and dissemination of scientific research documents, whether they are published or not. The documents may come from teaching and research institutions in France or abroad, or from public or private research centers.
L'archive ouverte pluridisciplinaire HAL, est destinée au dépôt et à la diffusion de documents scientifiques de niveau recherche, publiés ou non, émanant des établissements d'enseignement et de recherche français ou étrangers, des laboratoires publics ou privés. 


\title{
Numerical analysis of the V-Y shaped advancement flap
}

\author{
D. Remache $\mathrm{e}^{\mathrm{a}, \mathrm{b}, \mathrm{c}, 1,2}$, J. Chambert ${ }^{\mathrm{a}, \mathrm{b}, \mathrm{c}, *}$, J. Pauchot ${ }^{\mathrm{a}, \mathrm{d}, \mathrm{e}}$, E. Jacquet $\mathrm{t}^{\mathrm{a}, \mathrm{b}, \mathrm{c}}$ \\ ${ }^{a}$ University of Franche-Comté, 1 rue Claude Goudimel, 25030 Besançon Cedex, France \\ ${ }^{b}$ Department of Applied Mechanics, FEMTO-ST Institute - CNRS UMR 6174, \\ 24 rue de l'Épitaphe, 25000 Besançon, France \\ ${ }^{c}$ University Bourgogne Franche-Comté (UBFC) \\ ${ }^{d}$ Orthopedic, Traumatology, Aesthetic, Plastic, Reconstructive and Hand Surgery Unit, University Hospital of Besançon, 3 bd Alexandre Fleming, 25030 \\ Besançon, France \\ ${ }^{e}$ Research Unit, EA 4268 I4S IFR 133 INSERM, 25030 Besançon, France
}

\begin{abstract}
The V-Y advancement flap is a usual technique for the closure of skin defects. A triangular flap is incised adjacent to a skin defect of rectangular shape. As the flap is advanced to close the initial defect, two smaller defects in the shape of a parallelogram are formed with respect to a reflection symmetry. The height of the defects depends on the apex angle of the flap and the closure efforts are related to the defects height. Andrades et al. [1] have performed a geometrical analysis of the V-Y flap technique in order to reach a compromise between the flap size and the defects width. However, the geometrical approach does not consider the mechanical properties of the skin. The present analysis based on the finite element method is proposed as a complement to the geometrical one. This analysis aims to highlight the major role of the skin elasticity for a full analysis of the V-Y advancement flap. Furthermore, the study of this technique shows that closing at the flap apex seems mechanically the most interesting step. Thus different strategies of defect closure at the flap apex stemming from surgeon's know-how have been tested by numerical simulations.
\end{abstract}

Keywords: Human skin, V-Y advancement flap, Finite Element Method, Hyperelasticity

\section{Introduction}

Complex flaps such as the Limberg skin flap [2] or Z-plasty flap $[3,4]$ have been largely discussed in the literature on the contrast of the V-Y advancement flap for which only few theoretical studies have been proposed $[1,5]$. However this technique is generally used when a wound with a lack of substance needs to be covered.

A previous study [5] has been recently proposed to highlight the relation between the geometric parameters of the V-Y flap. In this previous paper, the skin has been considered as a rigid membrane and the technique has consisted in lessening the distance to suture through the three edges of the flap instead of concentrating on the initial lack of substance. Nevertheless the effective closure of skin defects is mainly obtained thanks to the elasticity of the tissue.

In the present paper, a more realistic approach is proposed by taking skin extensibility into account in order to quantify the sensitivity of several parameters for the V-Y advancement flap:

\footnotetext{
*Corresponding author. Tel.: +33 3816660 25; fax: +33 381666700

Email addresses: dj .remache@gmail.com (D. Remache), jerome. chambert@univ-fcomte.fr (J. Chambert),

julien.pauchot@gmail.com (J. Pauchot),

emmanuelle.jacquet@univ-fcomte.fr (E. Jacquet)

${ }^{1}$ Unité INSERM UMR 930 - Imagerie et Cerveau, Université François Rabelais, Équipe 5 - Imagerie et Ultrasons, 2 Bd Tonnellé, 37044 Tours Cedex 9, France.

${ }^{2}$ Laboratoire de Mécanique et Rhéologie, INSA Centre Val de Loire, Université François Rabelais Tours, 3 rue de la Chocolaterie CS 23410, 41034 Blois cedex, France.
}

- The dimensions of the flap ;

- The elastic properties of the skin ;

- The natural tension within the tissue ;

- The way to close and suture the wound.

The numerical analysis concerns the simulation of the suture and the prediction of the forces induced to close the wound. For each simulation, the skin flap is qualified by evaluating the closure effort at the most critical stitch point, also named the corner stitch. The critical stitch point is the one which requires the highest closure force and coincides with the flap apex when reaching the opposite edges of the wound. It is shown that a compromise between the size of the flap and the level of the closure effort value has to be found. The sensitivity to the mechanical parameters shows that the closure effort at the corner stitch is not only dependent on the size to be covered. At the end, the surgeon's technique is analysed: first by modifying the way of suturing the corner stitch and then by stretching the flap before suturing the wound.

\section{Clinical practice of the V-Y advancement flap}

\subsection{Description of the V-Y advancement flap}

In some clinical cases, it is necessary that the surgeon performs a chirurgical excision of cutaneous lesions such as tumours or nodules by cutting a large strip of skin. The V-Y 


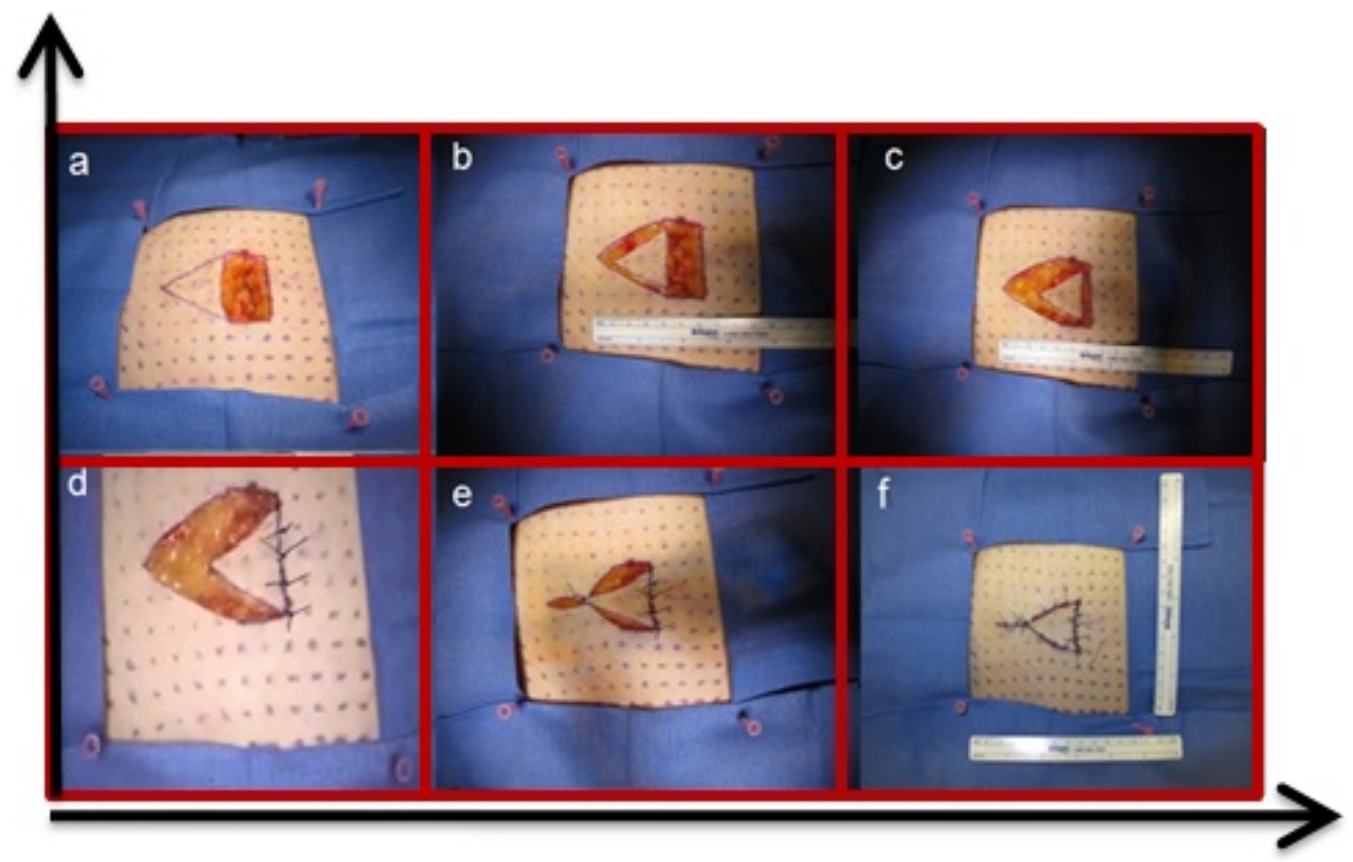

Figure 1: Successive steps of a V-Y advancement flap : (a) design of a triangular flap adjacent to a rectangular defect. (b) incision of the triangular flap. (c) translation of the flap. (d) three first stitches to fix the flap on the intact skin. (e) corner stitch at the flap apex. (f) final closure.

advancement flap is commonly used in practice to cover skin defect as illustrated in Fig. 1. The main interest of the V$\mathrm{Y}$ advancement flap lies in substituting the rectangular defect (Fig. 1a) adjacent to the base of the triangular flap by two smaller defects in the shape of parallelogram (Fig. 1d), which are adjacent to each side of the triangular flap. The main steps of this surgical technique are described as follows :

- First, the surgeon draws a rectangular pattern around the cutaneous lesion and an isosceles triangle adjacent to the largest dimension of rectangle. The dimensions of the triangle are defined by assessing the skin extensibility.

- Second, the surgeon excises the rectangular pattern, which takes the shape of a barrel (Fig. 1a) due to the natural tension of the skin. Indeed, the width of the rectangle in Fig. 1a is aligned with Langer's lines [6], which are parallel to the horizontal $x$-axis. Langer's lines define the direction within the human skin along which the skin exhibits minimum extensibility and maximum principal prestress. So, the skin natural tension is characterized by the orthogonal biaxial pre-stress state $\sigma_{x}$ and $\sigma_{y}$. By the way, the rectangular defect is stretched along the horizontal and vertical directions. The Langer's lines direction is of great interest for surgical operations in particular to obtain a better wound healing process.

- Third, the surgeon performs an complete incision of the isosceles triangle (Fig. 1b), which becomes a triangular flap submitted to skin contraction as a result of the skin pre-tension. The flap remains attached to the body and is always kept by a vascular supply.

- Fourth, in order to cover the defect, the flap (Fig. 1b) is submitted to an horizontal translation to reach the distal border (Fig. 1c).

- Fifth, the flap is sutured to the surrounding skin for the closure of the wound firstly at the distal border : three first stitches shown on Fig. 1d.

- Sixth, the flap is sutured at the flap apex (Fig. 1e). The fourth stitch, also called the corner stitch, is the most highly constrained.

- Finally, the whole flap is entirely sutured (Fig. 1f).

In current practice, the surgeon not only considers that the height of the flap remains unchanged before and after excising the triangular flap, but also performs a corner stitch with the nearest points on the opposite sides of the wound. Andrades et al. [1] have shown that once the dimensions of the defect are fixed, the angle at the flap apex and the flap height are the most important parameters of the flap geometry. These parameters depend on the location of the defect, the elasticity of the surrounding tissues and the cause of the surgery.

\subsection{Geometrical analysis of the $V-Y$ advancement flap}

As in the preliminary analysis [5], the lack of substance considered as rectangle $\left(B_{0} B_{0}^{\prime} C_{0}^{\prime} C_{0}\right)$ shown in Fig. 2 is covered by 


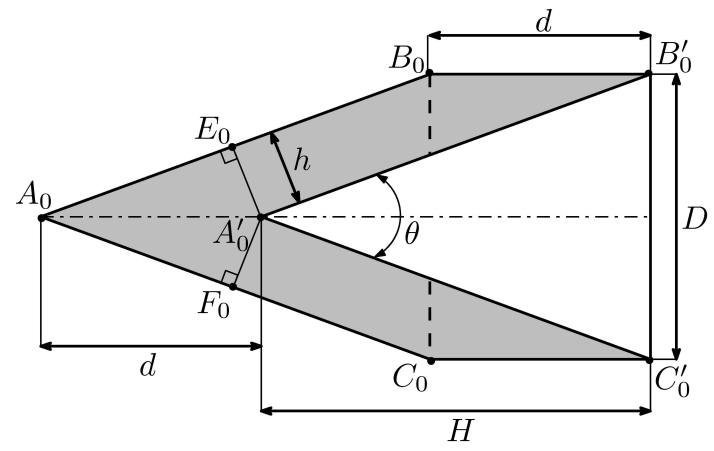

Figure 2: Geometrical description of the V-Y advancement flap.

a triangular flap $\left(A_{0} B_{0} C_{0}\right)$ incised from the largest dimension of the rectangle and translated through the defect $\left(A_{0}^{\prime} B_{0}^{\prime} C_{0}^{\prime}\right)$.

As described in Fig. 2, the dimensions of the lack of substance are $d \times D$. The angle at the flap apex is named $\theta$. The height of the flap is $H$. In the V-Y technique, the initial rectangular lack of substance is replaced by two parallelograms $\left(A_{0} A_{0}^{\prime} B_{0}^{\prime} B_{0}\right)$ and $\left(A_{0} A_{0}^{\prime} C_{0}^{\prime} C_{0}\right)$ of height $h$ which are formed by reflection symmetry (Fig. 2). The height of each parallelogram is smaller than the length of the initial rectangular one. The two following relations highlight the link between on the one hand $\theta$ and $h$, and on the other hand $\theta$ and $H$ [5]:

$$
\begin{gathered}
n=\frac{h}{d}=\sin \left(\frac{\theta}{2}\right) \\
\frac{H}{D}=\frac{1}{2 \tan \left(\frac{\theta}{2}\right)}
\end{gathered}
$$

The geometrical approach considers the skin as a rigid material moving in a horizontal plane and introduces a relationship between the angle $\theta$ and the dimensions of the parallelogram which needs to be covered by the V-Y advancement flap. Besides, flaps are generally designed with a $\theta$-angle of $30^{\circ}$ in order to reduce the depth of the area to suture and not to increase the width of the flap too much. But a geometrical analysis is not able to take into account the elasticity of the skin tissue while the mechanical extensibility is one of the main factors of the surgery success.

\section{Numerical modelling of the V-Y advancement flap}

\subsection{Finite element analysis of the $V-Y$ advancement flap}

In order to consider the elastic properties of the cutaneous tissue, a numerical analysis of the V-Y advancement flap is performed based on the finite element method. The lack of substance is a $20 \mathrm{~mm}$ width and $40 \mathrm{~mm}$ length rectangular hole into a planar skin domain $(470 \mathrm{~mm} \times 270 \mathrm{~mm})$. After excising the V-shape flap, the translation (from Fig. 1a to 1b) does not need a great force and the 3 first stitches are made for the flap and the surrounding skin to be joined (Fig. 1c). To simulate the step from Fig. 1d to 1e, the geometric domain is designed in Fig. 3a.

The skin is considered as a homogeneous, isotropic incompressible, and hyperelastic material. The constitutive law is an
Ogden (order 1) strain energy density function [7]. This model is a classical model used for non-linear complex materials such as rubber and biological tissues behaviour analysis. The strain energy function is given by :

$$
\Psi=\frac{2 \mu}{\alpha^{2}}\left(\lambda_{1}^{\alpha}+\lambda_{2}^{\alpha}+\lambda_{3}^{\alpha}-3\right)
$$

where $\mu$ the shear modulus under infinitesimal straining; $\alpha$ is a strain hardening exponent; and $\lambda_{1}, \lambda_{2}$ and $\lambda_{3}$ are the three principal stretch ratios.

In this study the planar non-linear finite element models of V-Y advancement flap have been performed with Ansys ${ }^{\circledR}$ v12 software. The skin sheet is meshed with 6-node triangular elements with plane stress hypothesis (PLANE183). The wound is considered to be small compared to the size of the skin sheet, and the mesh is refined in the vicinity of the flap.

As shown in Fig. 3, the symmetry of the problem is taken into account and only one half of the domain is modelled. The axis of symmetry is the horizontal $x$-axis which is approximately aligned with Langer's lines [6]. The symmetric boundary conditions impose null prescribed displacement in the vertical $y$ direction along the bottom side of the skin domain (see Fig. 3c and $3 \mathrm{e}$ ).

Naturally, the skin is under pretension. In order to simulate this pre-stress state, a biaxial-stress loading is applied to the initial mesh (Fig. 3b): $\sigma_{x}$ on the right side and $\sigma_{y}$ on the top side of the skin domain. In order to avoid rigid-body movements which could lead to numerical problems in the resolution by the finite element method, null displacements in the horizontal $x$-direction are imposed along the left side of the skin domain (see Fig. 3c and 3e).

For this study, the skin thickness is arbitrary fixed to $1 \mathrm{~mm}$. This value corresponds to that of the dermis which varies between 1 and $4 \mathrm{~mm}$ [8]. Knowing that it is the dermis which gives the skin its mechanical behaviour thanks to the collagen fibres located therein [9].

The proposed finite element simulation of the V-Y advancement flap is decomposed into two consecutive stages (see Fig. 3):

(i) The first stage consists in applying a biaxial-stress loading to the initial mesh (Fig. 3b). So the domain is deformed and new dimensions of the defect and the flap are shown in Fig. 3c and 3d. This deformed mesh is considered as the beginning of the second stage by keeping the stress and strain fields. The initial height of the defect $h$ becomes $h^{\prime}$ and the location of the flap apex is now $A_{1}^{\prime}$ (assimilated from now to the flap apex node) and its orthogonal projection on the opposite edge of the defect is $E_{1}$.

(ii) The second stage consists in applying a prescribed displacement to simulate the wound closure. As illustrated in Fig. $3 e$, the node $A_{1}^{\prime}$ located at the flap apex is now constrained to zero displacement in horizontal and vertical directions. Then, the node to be joined with the flap apex is chosen as the nearest of point $E_{1}$ and moved to its target. For simplification, $E_{1}$ is used for this node too. The resulting closure force at the node $E_{1}$ is calculated. 

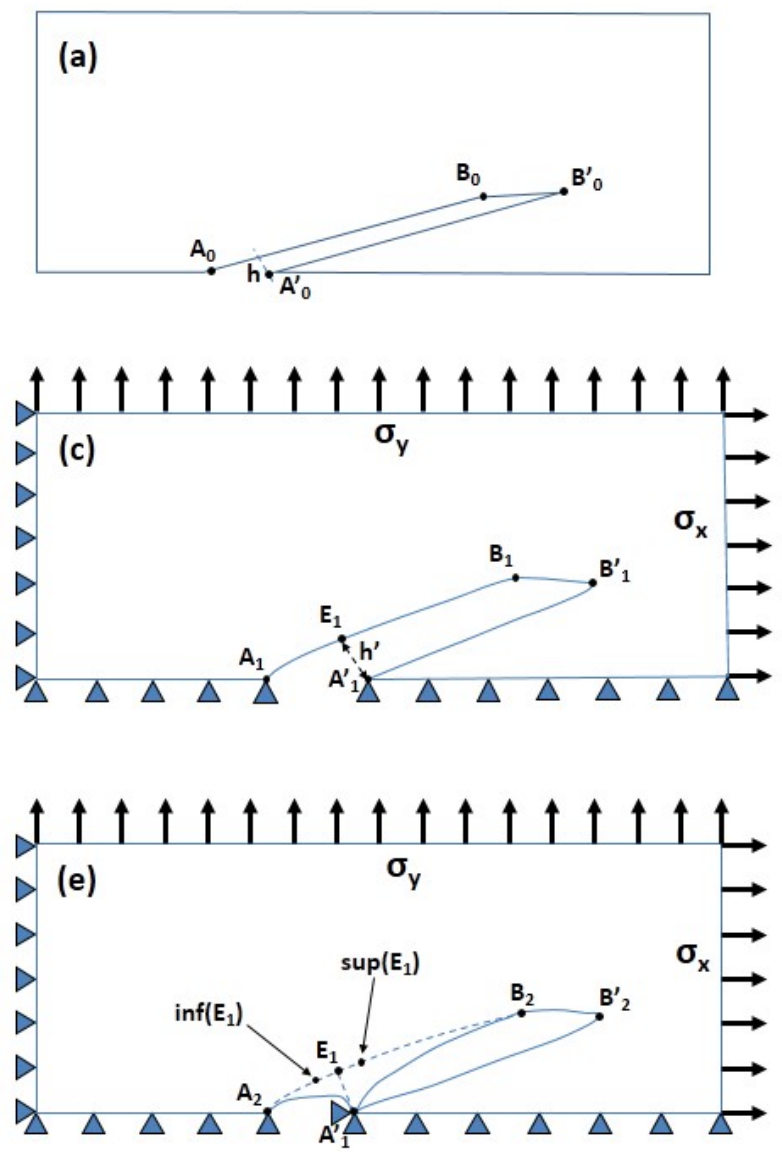
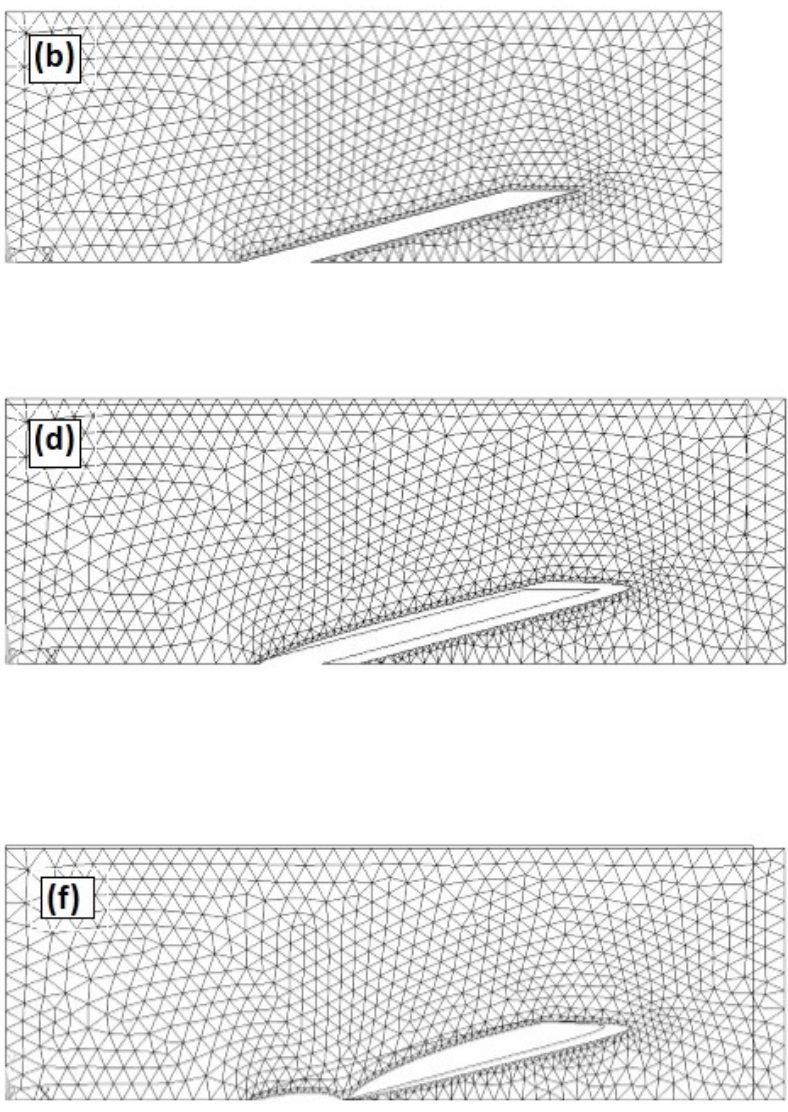

Figure 3: Numerical modelling for the suture of the corner stitch with $\theta=30^{\circ}$ : (a) edge of initial mesh. (b) initial mesh. (c) edge of pre-stressed domain with external boundary conditions. (d) deformed mesh of pre-stressed domain with the edge of initial mesh. (e) edge of final deformed mesh with external boundary conditions and the prescribed displacement for corner stitch closure. (f) final deformed mesh with the edge of pre-stressed domain.

\subsection{Parameters of the numerical simulation}

First a reference calculation is introduced before the description of the qualified procedures. The reference calculation includes a specific geometrical flap, the material properties and the initial pre-stress taking into account the natural tension of the in vivo skin. Shergold et al. [10] have fitted the Ogden model to uniaxial tensile test on abdomen ex vivo human skin performed by Jansen and Rottier [11]. The values of parameters $\alpha$ and $\mu$ obtained by Shergold et al. [10] are listed in Table 1 and correspond to material called Skin (1).

The reference values of the biaxial pre-stress field, entitled Pre-stress (1), are arbitrary chosen (see Table 2). The pre-stress field is characterized by the loading level (von Mises stress or equivalent tensile stress $\sigma_{\text {eq }}^{(1)}$ ) and the biaxiality ratio $r$ defined as : $r=\sigma_{y} / \sigma_{x}[12]$.

The reference case is defined as follows :

- Geometry: $\theta=30^{\circ}$;

- Material: Skin (1) in Table 1;

- Pre-loading: Pre-stress (1) in Table 2.
The modelling has been conducted both with this reference case and other ones in order to evaluate the influence of the angle, the material (Table 1) and the pre-stress state (Table 2). These parameter values of the reference case are assumed to represent the behaviour of the Skin (1) with the Pre-stress (1).

\begin{tabular}{lll}
\hline Material $(i)$ & $\mu^{(i)}(\mathrm{MPa})$ & $\alpha^{(i)}$ \\
\hline Skin $(1)$ & 0.11 & 9 \\
Skin $(2)$ & 0.03 & 13.46 \\
\hline
\end{tabular}

Table 1: Material parameters of the Ogden model for the considered skin.

\begin{tabular}{lllll}
\hline Pre-loading $(i)$ & $\sigma_{x}^{(i)}(\mathrm{kPa})$ & $\sigma_{y}^{(i)}(\mathrm{kPa})$ & $\sigma_{\mathrm{eq}}^{(i)}(\mathrm{kPa})$ & $r^{(i)}$ \\
\hline Pre-stress $(1)$ & 5.5 & 1 & 5.1 & 5.5 \\
Pre-stress $(2)$ & 30 & 8 & 26.9 & 3.75 \\
\hline
\end{tabular}

Table 2: Loading parameters for the considered pre-stress states.

\subsubsection{Variation of the angle}

For a rectangular defect defined ( $D$ and $d$ are fixed), the closure force is computed for eight flap apex angle values ranging from $20^{\circ}$ to $90^{\circ}$. When reducing the apex angle value, the 
height of the defect to close reduces too but the flap height increases (Fig. 4).

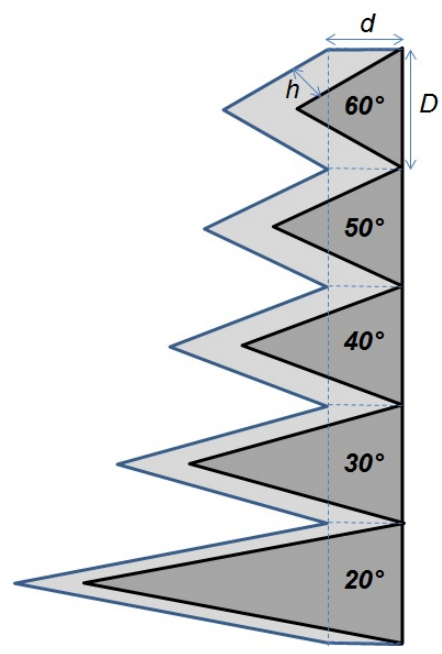

Figure 4: Different flap dimensions for various $\theta$-angles from $20^{\circ}$ to $60^{\circ}$.

The height of the final defect surrounding the flap varies between $3 \mathrm{~mm}$ and $14 \mathrm{~mm}$ and shows that for a value of $\theta$ equal to or greater than $60^{\circ}$, the suture distance at the apex of the flap is greater than the width $d$ of the initial rectangular defect. The closure force is calculated for each different geometry of the flap. Note that several angle values are not realistic. For instance when angle is upper than $60^{\circ}$, the length of the cumulative defect becomes upper than the initial defect one.

\subsubsection{Variation of the material behaviour}

In order to evaluate the results sensitivity to material properties, a second material is chosen. It is referenced as Skin (2) and two materials are tested (Table 1). The Ogden parameters of the second material are identified from ex vivo test performed on abdominoplasty human skin with a specific device [13], for which both forces and displacements of the tissue are registered at the same time. In this paper, the anisotropy has not been taken into account within the behaviour law.

Fig. 5a shows the stress-stretch ratio law of both materials. According to [14], it can be described by 3 consecutive stages. The first one is that in which the stress remains at a low level (Fig. 5b) and the skin loses its pleats, the fibres progressively change their orientation to be aligned with the loading direction (Fig. 5a). In the second one, the stress increases more fastly with stretch, and the stress-stretch ratio law becomes linear in the third one.

\subsubsection{Variation of the pre-stress state}

The sensitivity of the closure force is evaluated for two prestress loading levels illustrated in Table 2. A second set of biaxial pre-stress field, entitled Pre-stress (2), is identified by using MIC2M software developed by [15] and experimental results from [16]. The inverse identification procedure used in this software consists in choosing the pre-stress field which minimizes, according to Levenberg-Marquardt algorithm [17, 18], the difference between the predicted deformed rectangular defect and the real experimental one when the pre-stress is applied (Fig. 1a). The values of the biaxial pre-stress field are Prestress (2). These pre-stress states connect with the first stage of the stress-stretch ratio law. As described in [19], the skin state remains in regions in which strains are relatively small and stresses increase very slowly as strains increase. At these loading levels the Skin (1) is stiffer than Skin (2) (Fig. 5b).

\section{Numerical results}

The sensitivity analysis results are presented for various apex angle values by changing first the material properties, second the pre-stress loading. Third, the results concern the choice of the corner stitch and last the influence of different preliminary flap extensions.

\subsection{Influence of material properties}

The two different skins have been loaded under the reference Pre-stress (1) and the results are given in Fig. 6. In agreement with the geometric analysis of V-Y flap [1], the height of the skin defect initially pre-stressed, increases with the flap apex angle (Fig. 7).

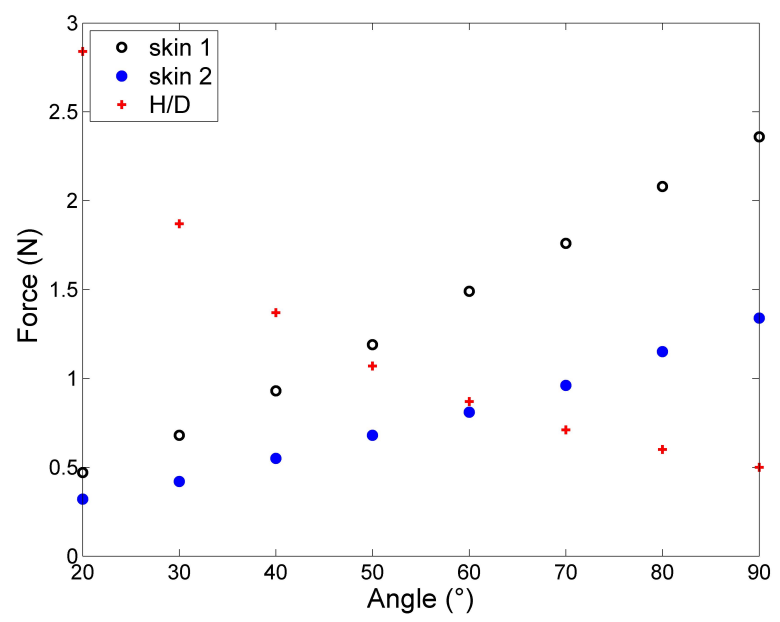

Figure 6: Closure force and ratio $H / D$ versus $\theta$-angle for Skin (1) and Skin (2) (Table 1) constrained to the reference Pre-stress (1) (Table 2).

Fig. 7 shows the relationship between the flap apex angle value and the distance to be covered which is linked to the adimensional ratio $n$ defined in equation 1 . Note that $n$ is exactly defined by $n=h / d$ in the geometric model and extrapolated by $n=h^{\prime} / d^{\prime}$ where $h^{\prime}$ and $d^{\prime}$ are respectively the height of the parallelogram defect and the width of the initial defect just after applying the pre-stress state. The Skin (1) and Skin (2) are pre-stretched to the reference pre-stress.

The obtained results show that for both material parameters sets, the closure force increases with the flap apex angle (Fig. 6), as the distance to cover (Fig. 7). The closure length in Skin (1) is smaller than the Skin (2)'s one (Fig. 7). As shown in Fig. 5b, the higher stiffness of Skin (1) in the low stress range 


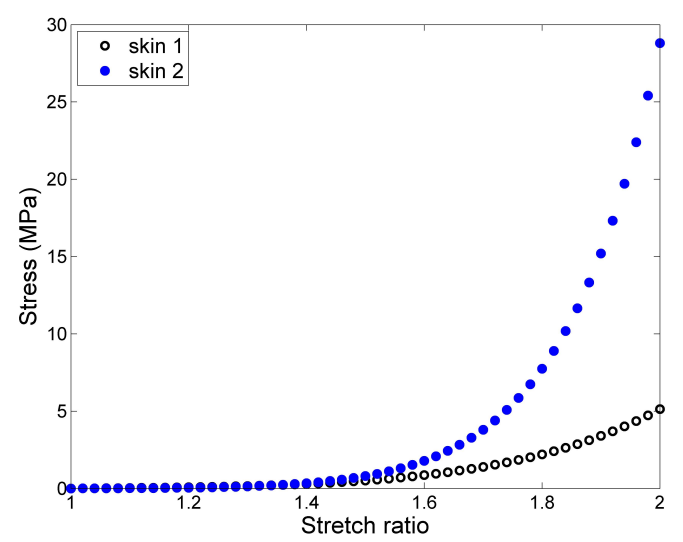

(a) Global view.

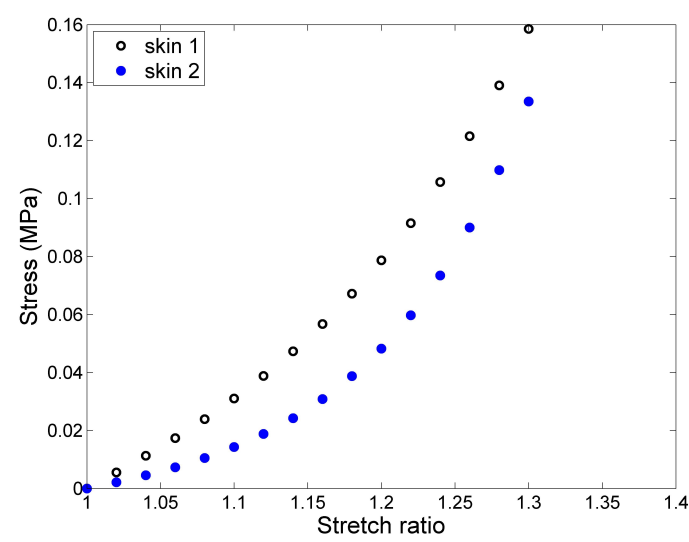

(b) Zoom in on low stretch ratio zone.

Figure 5: Behaviour laws of Skin (1) and Skin (2) (Table 1).

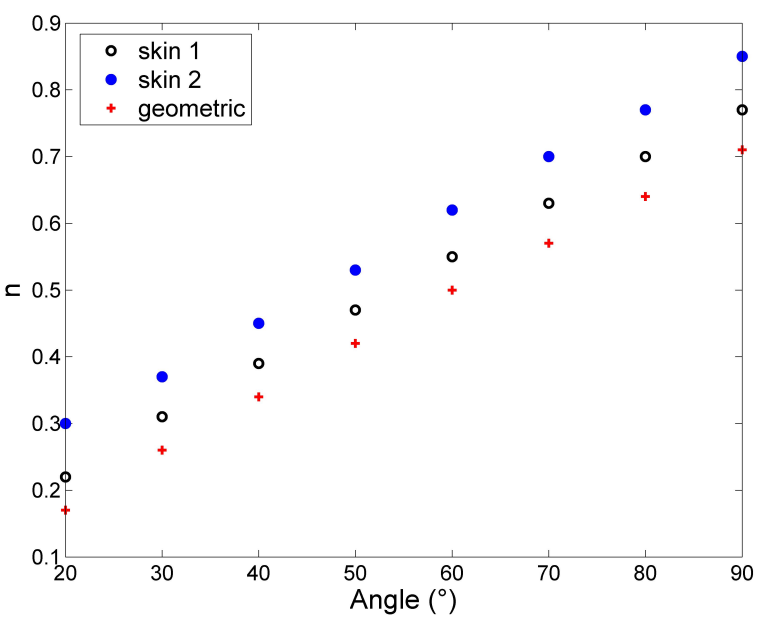

Figure 7: Adimentional ratio $n$ versus $\theta$-angle for Skin (1) and Skin (2) (Table 1) submitted to the reference Pre-stress (1) (Table 2), and for the rigid flap (geometric).

value leads to a smaller closure force than Skin (2)'s one despite that the adimensional distance to cover is greater for Skin (2) (Fig. 7).

\subsection{Influence of the pre-stress state}

The two different pre-stresses in Table 2 have been applied to Skin (1) and the results are given in Fig. 8. The closure force at the flap apex increases with the flap apex angle and hence the height of the skin defect. It confirms that the resistance of the flap to wound closure increases when the height of the flap decreases.

\subsection{Choice of the corner stitch}

The calculation for choosing the corner stitch which is the most loaded one, is done with the Skin (2) materials parameters and the Pre-stress (1) state. As shown in Fig. 3, by defining $E_{1}$ as the orthogonal projection of flap apex location $A_{1}^{\prime}$ onto the

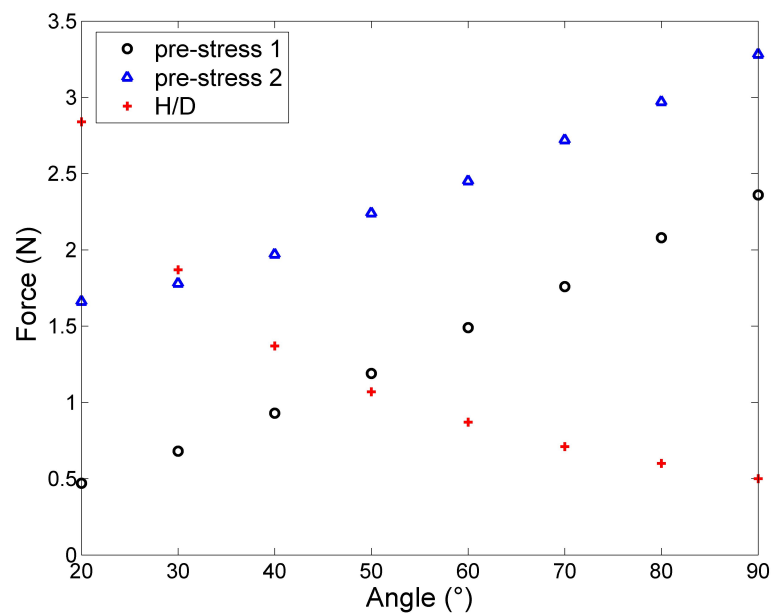

Figure 8: Closure force and ratio $H / D$ versus $\theta$-angle for a unique skin parameters set Skin (1) (Table 1) submitted to Pre-stress (1) and Pre-stress (2) (Table 2).

opposite edge, the closure force is computed at different nodes in the vicinity of $E_{1}$ that is to say between the extrema points $\inf \left(E_{1}\right)$ and $\sup \left(E_{1}\right)$. The algebraic distance $\mathrm{d}\left(E_{1}, M\right)$ between a variable point $M$ and the defined point $E_{1}$ is taken down and carried forward as a negative value where $M$ is nearest to $\inf \left(E_{1}\right)$ and a positive value where $M$ is nearest to $\sup \left(E_{1}\right)$.

$$
\mathrm{d}\left(E_{1}, M\right)= \pm \sqrt{\left(x_{M}-x_{E_{1}}\right)^{2}+\left(y_{M}-y_{E_{1}}\right)^{2}}
$$

where $\left(x_{M}, y_{M}\right)$ and $\left(x_{E_{1}}, y_{E_{1}}\right)$ are respectively the cartesian coordinates of the points $M$ and $E_{1}$. The algebraic distance is taken positive if $M$ belongs to the segment $\left[E_{1} ; \sup \left(E_{1}\right)\right]$ and negative if $M$ belongs to the segment $\left[\inf \left(E_{1}\right) ; E_{1}\right]$.

In order to automatically find the "optimal" corner stitch for different flap geometries ( $\theta$-angle in the $20^{\circ}-60^{\circ}$ range), a numerical study has been carried out by coupling Ansys ${ }^{\circledR}$ and Matlab $^{\circledR}$ softwares [20]. A parametric modelling was necessary to determine automatically the location of the nodes to be 


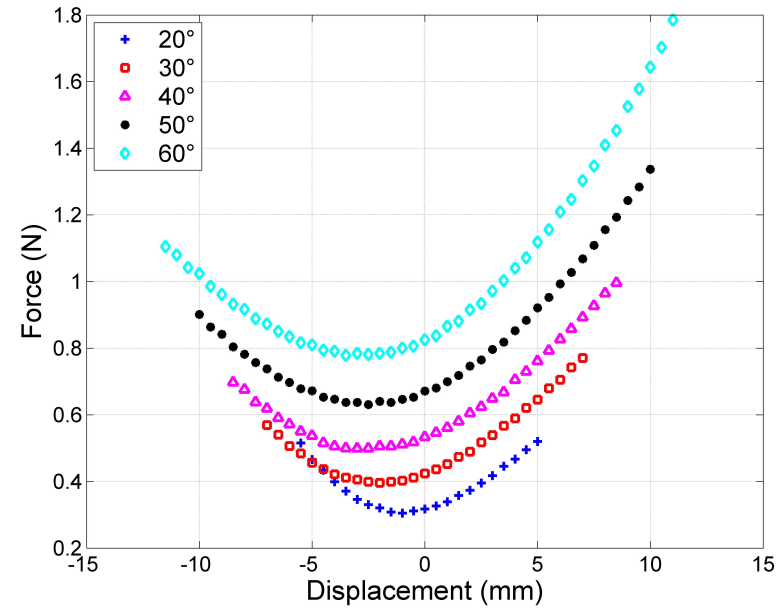

Figure 9: Closure force needed to join the different nodes in the vicinity of $E_{1}$ to $A_{1}^{\prime}$ versus distance $\mathrm{d}\left(E_{1}, M\right)$ for different geometries $\left(\theta\right.$-values from $20^{\circ}$ to $60^{\circ}$ ) with Skin (2) (Table 1) and Pre-stress (1) (Table 2).

moved to the flap apex.

The influence of the closure node choice has been numerically analyzed. For various angles, Fig. 9 shows the closure force needed to join separately the different nodes close to $E_{1}$ with the node $A_{1}^{\prime}$ versus the algebraic distance d $\left(E_{1}, M\right)$. Fig. 9 highlights minimum values of the closure force for each various curve corresponding to each various angle in the segment $\left[\inf \left(E_{1}\right) ; E_{1}\right]$.

It appears that the minimal force $F_{\min }$ is not equal to the force obtained when joining $E_{1}$ with $A_{1}^{\prime}$ but, at a stitch node $M_{\text {min }}$ located at about a few millimetres forward from $E_{1}$. The force obtained when joining $E_{1}$ (respectively $M_{\min }$ ) with $A_{1}^{\prime}$ is named $F_{\text {orth }}$ (respectively $F_{\text {min }}$ ).

The force values $F_{\text {orth }}, F_{\text {min }}$, the relative difference $\Delta F / F_{\text {orth }}$ and the distance $\mathrm{d}\left(E_{1}, M_{\min }\right)$ are reported in Table 3 . The force relative gain provides a real comfort to patient by reducing of a few percent the amplitude of the force necessary to close the wound.

\begin{tabular}{lccccc}
\hline$\theta\left(^{\circ}\right)$ & 20 & 30 & 40 & 50 & 60 \\
\hline$h(\mathrm{~mm})$ & 3.47 & 5.17 & 6.84 & 8.45 & 9.23 \\
$h^{\prime}(\mathrm{mm})$ & 5.93 & 7.37 & 9.02 & 10.68 & 12.33 \\
$F_{\text {orth }}(\mathrm{N})$ & 0.32 & 0.42 & 0.54 & 0.67 & 0.83 \\
$F_{\text {min }}(\mathrm{N})$ & 0.31 & 0.39 & 0.50 & 0.63 & 0.78 \\
$\Delta F / F_{\text {orth }}(\%)$ & 3.13 & 7.14 & 7.41 & 5.97 & 6.02 \\
$\mathrm{~d}\left(E_{1}, M_{\min }\right)(\mathrm{mm})$ & -1. & -2. & -2.5 & -2.5 & -2.5 \\
\hline
\end{tabular}

Table 3: Summary results showing the force gain corresponding to the choice of the critical suture node in preferably to orthogonal projection of the apex flap $\left(\theta=30^{\circ}\right.$, Skin (1) and Pre-stress (1)).

\subsection{Influence of horizontal extension of the flap}

In this section, the reference configuration is chosen $(\theta=$ $30^{\circ}$, Skin (1) and Pre-stress (1)). The modelling phases are as follows :

- First, as previously, the biaxial pre-stress field is applied so that the flap apex moves from the initial position $A_{0}^{\prime}$ (trian-

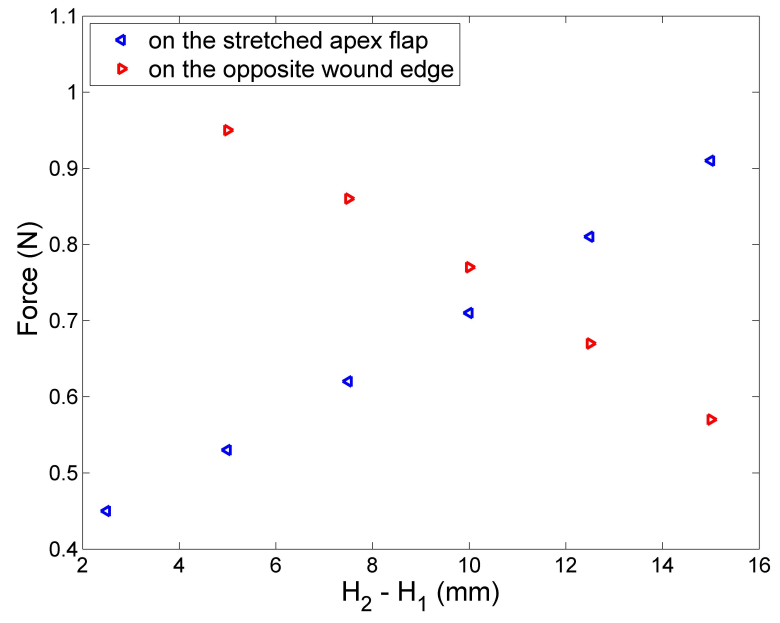

Figure 11: Force $F\left(A_{2}^{\prime}\right)$ needed to stretch the flap in left triangle symbol and resulting closure force $F\left(E_{2}\right)$ needed to join $E_{2}$ with $A_{2}^{\prime}$ in right triangle symbol versus stretch increment $H_{2}-H_{1}\left(\theta=30^{\circ}\right.$, Skin (1) and Pre-stress (1)).

gle initial height $H_{0}$, Fig. 10a) to the position $A_{1}^{\prime}$ (triangle height $H_{1}$, Fig. 10b) ;

- Second, the flap apex is stretched lengthwise in the horizontal axis direction from the position $A_{1}^{\prime}$ (Fig. 10b) to the position $A_{2}^{\prime}$ (triangle height $H_{2}$, Fig. 10c). Thus the stretch increment is $\left\|\overrightarrow{A_{1}^{\prime} A_{2}^{\prime}}\right\|=H_{2}-H_{1}$;

- Third, the node $A_{2}^{\prime}$ is constrained to zero displacement and the nearest node of the orthogonal projection of $A_{2}^{\prime}$ named $E_{2}$ (Fig. 10c) is moved towards $A_{2}^{\prime}$ to suture the fourth stitch (Fig. 10d). Then the closure force is calculated at node $E_{2}$;

- All these phases are repeated for several flap-stretching values from $2.5 \mathrm{~mm}$ to $15 \mathrm{~mm}$ with a $2.5 \mathrm{~mm}$-step. At each iterative calculation, the displacement of apex point $A_{1}^{\prime}$, that is to say $\left\|\overrightarrow{A_{1}^{\prime} A_{2}^{\prime}}\right\|=H_{2}-H_{1}$, is evaluated. Then the resulting forces are taken down first at the flap apex node $A_{2}^{\prime}$ and after at the node $E_{2}$ when joining $E_{2}$ from the opposite edge of the defect and $A_{2}^{\prime}$ at the flap apex.

Fig. 11 shows, on the one hand the extension force at the node $A_{2}^{\prime}$ (left triangle symbol) for several stretch increments $H_{2}-H_{1}$, on the other hand the resulting force at the node $E_{2}$ (right triangle symbol) for the closure. The extension force at $A_{2}^{\prime}$ named $F\left(A_{2}^{\prime}\right)$ increases according to the flap stretch amplitude, while the closure resulting force at $E_{2}$ named $F\left(E_{2}\right)$ decreases. However, it should be noticed that force directions are not the same for both steps.

\section{Discussion}

The numerical modelling of the V-Y advancement flap and its environment, taking into account the elastic behaviour of the skin and its initial tension, requires the closure force value which is a relevant information in order to prevent necrosis. In 

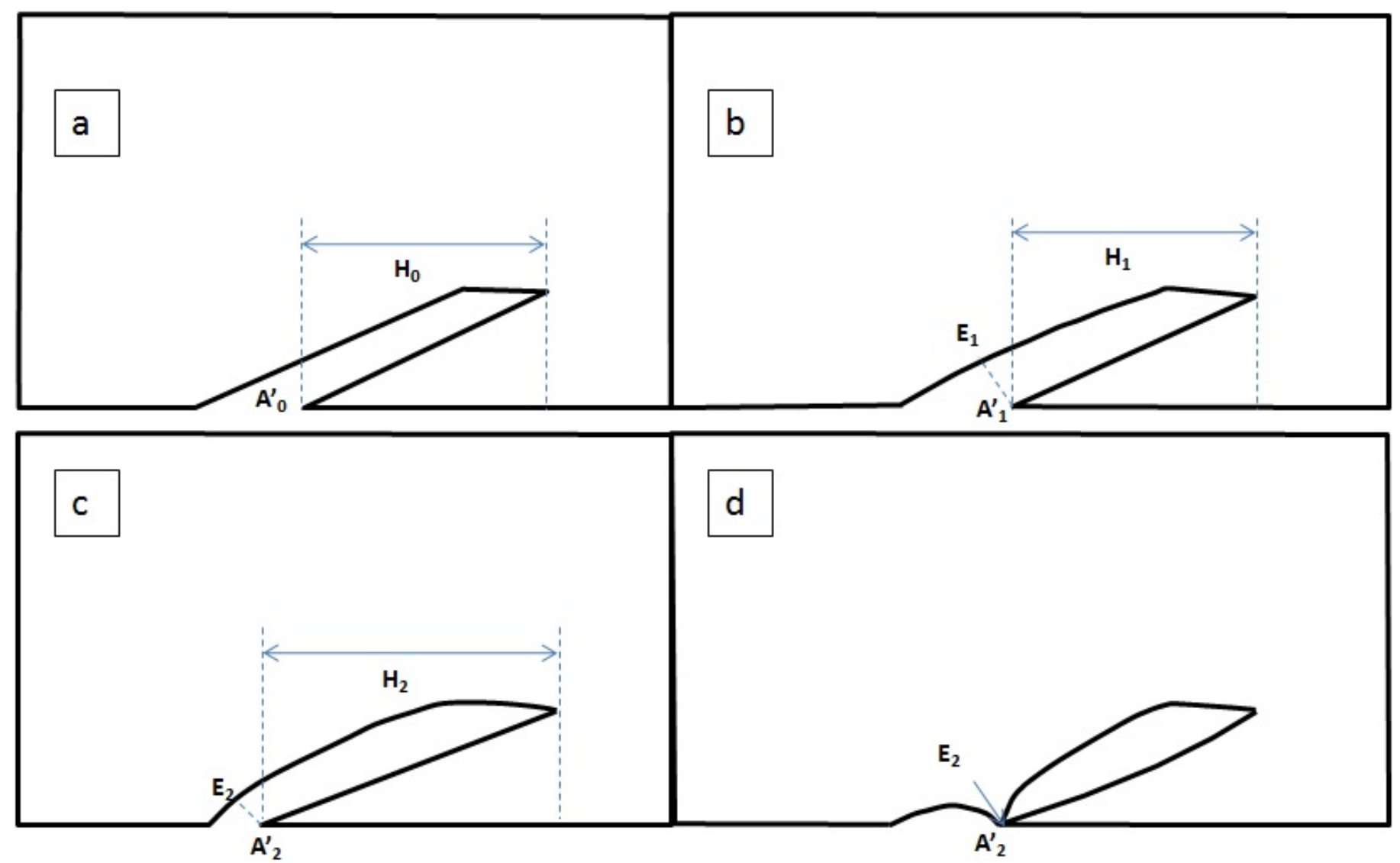

Figure 10: Numerical modelling of the horizontal flap extension for $\theta=30^{\circ}$, Skin (1) and Pre-stress (1) : (a) initial step. (b) intermediary step corresponding to pre-stressed domain. (c) horizontal stretch of the flap. (d) final step.

this study case for assumptions and data used, the simulation of the closure at the flap apex determines a closure force value between 0 and $3 \mathrm{~N}$ whatever the flap angle value is. The results depend on elastic parameters of the skin and value of initial biaxial loading component. As it was expected, the closure force is greater (i.e. the skin is more tight) if the flap angle value is larger or if the pre-stress field is higher (Fig. 8).

As regards the flap angle value, the results are consistent with the geometrical analysis of the V-Y advancement flap [5] by itself. On the contrary, it has been shown that the width of the skin defect to cover and the stiffness of the skin are not necessarily adequate to predict the closure force. Indeed, the non-linearity of the behaviour law provides Skin (1) stiffer than Skin (2) at low extension (below 1.35 stretch) and Skin (2) stiffer than Skin (1) at high extension (above 1.35 stretch) (Fig. 5).

From the surgeon point of view, V-Y advancement flap is a solution to the problem of wound with loss of substance. The surgeon's priority consists in covering and closing the loss of substance. In the case of a soft skin, the V-Y flap is chosen with a great angle flap value. In the case of a stiffer skin or a more tight skin, the surgeon designs a longer flap with an apex angle of about $20^{\circ}$ [21] to ensure closure without complication such as necrosis [22]. The surgeon fits the flap technique to the patient (localization of the wound, qualitative evaluation of mechanical properties and tension of the skin). The quantitative characterization of the specific-patient skin should be implemented to supply the numerical model which would help the surgery design.

Notice that the real behaviour of the skin is viscous hyperelastic. The modelization does not take into account the time effect. It is supposed that the initial value of closure force is maximal at the beginning of the suture, so the immediate response of the skin matches to the higher loaded skin. Nonetheless, the relaxation sensitivity of the model should be evaluated in a future work.

In addition, this study has shown that optimizing the choice of the stitch point allows to reduce the closure forces. For the parameters and assumptions done, the force gain is between 3.13 and $7.41 \%$. This optimal node is located at a point between $1 \mathrm{~mm}$ and $2.5 \mathrm{~mm}$ in front of the orthogonal projection $E_{1}$ of the flap onto the opposite side of the wound (Fig. 9). However, the optimization is not yet compared to experimental tests. The simulation is restricted to the corner stitch closure with a plane modelling by using an isotropic behaviour law without taking into account the viscoelasticity of the skin, nor its heterogeneity. The corner stitch is supposed to be the one that requires the maximal closure force of the V-Y technique.

Even if there is a good correlation between experimental tests and simulations, the results should be put into perspective because of mechanical variability and sensitivity to elastic param- 
eters. After qualitatively evaluating the specific-patient case and choosing the geometry and localization of the V-Y flap, the surgeon's practical experience consists in first stretching the flap and second performing a corner stitch point with the nearest point on the opposite side. The calculated "optimized" stitch is consistent with the surgeon's practise. The first stretching of the flap compensates for the retraction of the skin because of the in vivo natural stress. This stretching replaces the flap apex at its original position. Then the surgeon sutures the flap directly with the nearest point on the opposite edge of the wound. Quantitatively, the relative difference in closure force is able to be up to about $7 \%$ (see Table 3). This can be considered low but can bring comfort to the patient and possibly avoid a complication.

In Fig. 11, the stretch force is proportional to the stretch itself and the resulting closure force is inversely proportional to the stretch. The compromise between force of the stretch and the corresponding closure force is not easy to find but exists. The stretching of the flap reduces the width of the defect and thus reduces the closure force even if the stretch needs some effort too.

This is of crucial interest for the surgeon who tries to reduce the tension within the skin in order to avoid any risk of necrosis. Indeed, if a cutaneous necrosis occurs in the neighbourhood of the scar, the wound healing process is definitively stopped in the affected area. Finally, the numerical analysis of the V$\mathrm{Y}$ advancement flap by finite element method may give useful indication for the surgeon.

In conclusion, this finite element analysis of the V-Y advancement flap has highlighted the key role of skin elasticity for the choice of the flap apex angle. In addition two different versions of suture technique at the flap apex (with and without stretching the flap) are numerically evaluated. Both methods are aimed at minimizing the tension at the corner stitch, and are compared from a mechanical point of view. These two methods simulate the surgical gesture. Nevertheless, the mechanical aspect is only one among many other medical factors, and the final choice of the closure technique remains the surgeon's decision.

\section{Conflict of interest statement}

No conflict of interest.

\section{Funding and ethical approval}

Funding : none declared.

Ethical approval : not required.

\section{References}

[1] Andrades PR, Calderon W, Leniz P, Bartel G, Danilla S, Benitez S. Geometric analysis of the V-Y advancement flap and its clinical applications. Plast Reconstr Surg 2005;115(6):1582 - 1590.

[2] Lister GD, Gibson T. Closure of rhomboid skin defects: The flaps of Limberg and Dufourmentel. Br J Plast Surg 1972;25:300 - 314.

[3] Retel V, Vescovo P, Jacquet E, Trivaudey F, Varchon D, Burtheret A. Nonlinear model of skin mechanical behaviour analysis with finite element method. Skin Res Technol 2001;7(3):152-158.
[4] Chu DY. Mathematical principle of planar Z-plasty. Plast Reconstr Surg 2000;105(1): $105-108$.

[5] Pauchot J, Chambert J, Remache D, Elkhyat A, Jacquet E. Geometrical analysis of the V-Y advancement flap applied to a keystone flap. J Plast Reconstr Aesthet Surg 2012;65(8):1087-1095.

[6] Langer K. On the anatomy and physiology of the skin: I. The cleavability of the cutis. Br J Plast Surg 1978;31(1):3 - 8. (Translated by T. Gibson from Langer K. (1861). Zur Anatomie und Physiologie der Haut. I. Uber die Spaltbarkeit der Cutis. Sitzungsbericht der Mathematischnaturwissenschaftlichen Classe der Kaiserlichen Academie der Wissenschaften, Wien, 44, pp. 19-46).

[7] Ogden RW. Large deformation isotropic elasticity - on the correlation of theory and experiment for incompressible rubberlike solids. Proc R Soc Lond A 1972;326(1567):565 - 584.

[8] Xu F, Lu T. Introduction to Skin Biothermomechanics and Thermal Pain. Springer; 2011.

[9] Brown IA. A scanning electron microscope study of the effects of uniaxial tension on human skin. Br J Dermatol 1973;89(4):383 - 393.

[10] Shergold OA, Fleck NA, Radford D. The uniaxial stress versus strain response of pig skin and silicone rubber at low and high strain rates. Int $\mathbf{J}$ Impact Eng 2006;32(9):1384-1402.

[11] Jansen LH, Rottier PB. Some mechanical properties of human abdominal skin measured on excised strips: A study of their dependence on age and how they are influenced by the presence of striae. Dermatol 1958;117(2):65-83.

[12] Reihsner R, Balogh B, Menzel EJ. Two-dimensional elastic properties of human skin in terms of an incremental model at the in vivo configuration. Med Eng Phys 1995;17(4):304-313.

[13] Capek L, Lochman Z, Dzan L, Jacquet E. Biaxial extensometer for measuring of the human skin anisotropy in vivo. In: 5th Cairo International Biomedical Engineering Conference. IEEE, Cairo, Egypt; 2010, p. 83 85.

[14] Delalleau A, Josse G, Lagarde JM, Zahouani H, Bergheau JM. A nonlinear elastic behavior to identify the mechanical parameters of human skin in vivo. Skin Res Technol 2008;14(2):152-164.

[15] Richard F. Identification du comportement et évaluation de la fiabilité des composites stratifiés. Ph.D. thesis; University of Franche-Comté, Besançon, France; 1999.

[16] Remache D, Pauchot J, Chambert J, Capek L, Jacquet E. Experimental and numerical analysis of a V-Y advancement flap on human skin ex vivo. Comput Methods Biomech Biomed Eng 2011;14(sup1):137 - 138.

[17] Levenberg K. A method for the solution of certain non-linear problems in least squares. Quart Appl Math 1944;2(2):164-168.

[18] Marquardt DW. An algorithm for least-squares estimation of nonlinear parameters. J Soc Ind Appl Math 1963;11(2):431 - 441.

[19] Chaudhry HR, Bukiet B, Findley T, Ritter AB. Evaluation of residual stress in rabbit skin and the relevant material constants. J Theor Biol 1998;192(2): $191-195$.

[20] Billon K, Gallecier F. Numerical optimization of the V-Y advancement flap geometry. Technical report; University of Franche-Comté, Besançon, France; 2012.

[21] Pauchot J, Servagi S, Laveaux C, Lasserre G, Tropet Y. Bilateral latissimus dorsi V-Y musculocutaneous rotation flap for closure of a large dorsal radionecrosis. Geometric analysis and interest. About one case. Ann Chir Plast Esthét 2010;55(1):66-70.

[22] Pauchot J, Remache D, Chambert J, Elkhyat A, Jacquet E. Finite element analysis to determine stress fields at the apex of V-Y flaps. Eur J Plast Surg 2013;36(3):185 - 190 . 